\title{
Elevated serum estradiol levels in artificial autologous frozen embryo transfer cycles negatively impact ongoing pregnancy and live birth rates
}

\author{
Rani Fritz $^{1}$ (D) $\cdot$ Sangita Jindal $^{1} \cdot$ Heather Feil $^{1} \cdot$ Erkan Buyuk $^{1}$
}

Received: 30 May 2017 / Accepted: 1 August 2017 /Published online: 19 August 2017

(C) Springer Science+Business Media, LLC 2017

\begin{abstract}
Purpose The aim of this study is to evaluate the correlation between serum estradiol $\left(\mathrm{E}_{2}\right)$ levels during artificial autologous frozen embryo transfer (FET) cycles and ongoing pregnancy/live birth rates $(\mathrm{OP} / \mathrm{LB})$.

Methods A historical cohort study was conducted in an academic setting in order to correlate peak and average estradiol levels with ongoing pregnancy/live birth rates for all autologous artificial frozen embryo transfer cycles performed from 1/2011 to $12 / 2014$.

Results Average and peak $\mathrm{E}_{2}$ levels from 110 autologous artificial FET cycles from 95 patients were analyzed. Average $\mathrm{E}_{2}$ levels were significantly lower in cycles resulting in $\mathrm{OP} / \mathrm{LB}$ compared to those that did not $(234.1 \pm 16.6 \mathrm{pg} / \mathrm{ml} \mathrm{vs}$. $315 \pm 24.8 \mathrm{pg} / \mathrm{ml}$, respectively, $p=0.04$ ). Although peak $\mathrm{E}_{2}$ levels were not significantly different between cycles resulting in OP/LB compared with those that did not $(366.9 \pm 27.7 \mathrm{pg} /$ $\mathrm{ml}$ vs. $459.1 \pm 32.3 \mathrm{pg} / \mathrm{ml}$, respectively, $p=0.19)$, correlation analysis revealed a statistically significant $(p=0.02)$ downward trend in $\mathrm{OP} / \mathrm{LB}$ rates with increasing peak $\mathrm{E}_{2}$ levels. Conclusions This study suggests that elevated $\mathrm{E}_{2}$ levels in artificial autologous FET cycles are associated with lower $\mathrm{OP} / \mathrm{LB}$ rates. Estradiol levels should be monitored during artificial FET cycles.
\end{abstract}

Keywords Estradiol $\cdot$ In vitro fertilization · Frozen embryo transfer cycles $\cdot$ Live birth rates

Erkan Buyuk

erbuyuk@yahoo.com

1 Department of Obstetrics \& Gynecology and Women's Health, Albert Einstein College of Medicine/Montefiore Medical Center, 1300 Morris Park Ave Block 634, Bronx, NY 10461, USA

\section{Introduction}

Frozen non-donor embryo transfer cycles account for approximately $24.5 \%$ of total IVF cycles reported to the CDC in 2013 [1]. The percentage of frozen embryo transfer (FET) cycles is dramatically increasing, in part due to a trend to transfer fewer embryos, improved cryopreservation techniques, and elective oocyte cryopreservation [2]. Additionally, recent evidence suggests that pregnancy rates following in vitro fertilization (IVF) could be improved with elective FET cycles in place of fresh embryo transfers [3-5]. Frozen embryo transfer cycles, either natural or artificial, require synchronization of the endometrium with development of the embryo. Natural FET cycles rely on growth of a dominant follicle with subsequent endometrial maturation by endogenous estradiol $\left(\mathrm{E}_{2}\right)$ and progesterone. Artificial FET cycles commonly rely on suppression of the hypothalamic-pituitary-ovarian axis with administration of exogenous $E_{2}$ and progesterone for endometrial/ embryo synchronization.

Successful implantation and subsequent healthy pregnancy are dependent on invasion of embryo-derived trophoblast cells through the maternal decidua and myometrium, with eventual remodeling of the myometrial spiral arteries [6]. This process is regulated by a complex interaction between maternal and fetal tissues, mediated by numerous cytokines, growth factors, and peptides [7]. Estradiol is critical to endometrial and placental development $[8,9]$; however, excess $\mathrm{E}_{2}$ in the early stages of pregnancy can have adverse effects on placentation. Elevated $\mathrm{E}_{2}$ has been shown to adversely affect endometrial receptivity [10] and elevated levels in fresh cycles prior to transfer are correlated with decreased implantation rates [11, 12]. Additionally, elevated $E_{2}$ levels during IVF cycles have been linked to an increased incidence of adverse pregnancy outcomes including pre-eclampsia and intrauterine growth restriction $[13,14]$. 
Estradiol levels are a modifiable variable in artificial FET cycles. The aim of this study is to evaluate the impact of peak and average $E_{2}$ concentrations prior to progesterone administration in autologous artificial FET cycles on ongoing pregnancy/live birth rates $(\mathrm{OP} / \mathrm{LB})$.

\section{Materials and methods}

A total of 110 artificial autologous FET cycles from 95 patients with a subsequent embryo transfer were conducted from 1/2011 to 12/2014 at Montefiore's Institute for Reproductive Medicine and Health. Inclusion criteria included patients undergoing an artificial autologous FET cycle primarily with transdermal or intramuscular $\mathrm{E}_{2}$ supplementation with or without the addition of vaginal $\mathrm{E}_{2}$. Exclusion criteria included natural FET cycles, donor FET cycles, and initiated artificial cycles without a subsequent embryo transfer. This study was approved by the Albert Einstein College of Medicine Institutional review board (IRB number 2016-6057). Data were extracted including patient's age, duration of FET cycle, maximum historical serum follicle stimulating hormone (FSH) level, route of insemination, gravidity, parity, body mass index (BMI), race, route of $E_{2}$ administration, use of vaginal $E_{2}$ supplementation, maximal endometrial stripe (EMS) during the FET cycle, route of progesterone administration, serum $\mathrm{E}_{2}$ levels from initiation of the FET cycle to levels immediately prior to progesterone supplementation, peak $E_{2}$ levels, average $E_{2}$ levels, day of embryo transfer, number of embryos transferred, and whether the transfer resulted in a biochemical pregnancy, clinical pregnancy, ongoing pregnancy/live birth, or spontaneous abortion. Biochemical pregnancy was defined as positive $\beta$ human chorionic gonadotropin ( $\beta$ hCG) level without evidence of a gestational sac on ultrasound. Clinical pregnancy was defined as evidence of a gestational sac on ultrasound. Ongoing pregnancy was defined as intrauterine pregnancy with a heart beat at 20 weeks or later. Peak serum $\mathrm{E}_{2}$ levels were considered the highest serum $\mathrm{E}_{2}$ level $(\mathrm{pg} / \mathrm{ml})$ obtained from initiation of the artificial FET cycle to the level immediately prior to progesterone supplementation, whereas average serum $\mathrm{E}_{2}$ levels (pg/ $\mathrm{ml}$ ) were the average of all serum $\mathrm{E}_{2}$ levels recorded from the initiation of the artificial FET cycle to the last level prior to progesterone supplementation.

\section{Stimulation protocol}

Frozen embryo transfer cycles were started either following hormonal suppression with oral contraception, GnRH agonist (leuprolide acetate) suppression, combination of leuprolide acetate, and hormonal suppression, or no suppression. Following confirmation of suppressed $\mathrm{E}_{2}$ levels (defined as serum $\mathrm{E}_{2}$ level below $50 \mathrm{pg} / \mathrm{ml}$ ) and a negative $\beta$ hCG test, supplemental $\mathrm{E}_{2}$ was initiated primarily transdermally $(100 \mathrm{mcg} / \mathrm{patch})$ every other day or intramuscularly $(2 \mathrm{mg})$ twice per week. Trough $\mathrm{E}_{2}$ levels were measured twice weekly for at least 2 weeks and $\mathrm{E}_{2}$ dosage was adjusted accordingly to achieve an $E_{2}$ level of 200-500 pg/ml following 8-10 days of $\mathrm{E}_{2}$ supplementation. Progesterone supplementation was added once adequate $\mathrm{E}_{2}$ levels were achieved, and an adequate EMS was appreciated by ultrasound ( $\geq 7 \mathrm{~mm}$ ). Progesterone was either administered in oil intramuscularly $(50 \mathrm{mg})$ daily or vaginally via Endometrin (Ferring Pharmaceuticals, Parsippany, NJ) at $100 \mathrm{mg}$ three times a day or Crinone (Allergan, Irvine, CA). Estradiol was measured in $\mathrm{pg} / \mathrm{ml}$ using Immulite 2000 (Siemens, Munich, Germany). Embryo transfer was performed under ultrasound guidance on the 5th day of progesterone administration for a day 3 embryo transfer and on the 7th day of progesterone administration for a day 5 embryo transfer.

\section{Statistical analysis}

Data are presented as mean \pm standard error of the mean (SEM). Student's $t$ test or Mann Whitney U test (if data were not normally distributed) were used to compare the demographic and cell cycle characteristics that are characterized with continuous data. Chi square or Fisher exact tests were used for categorical data. Logistic regression on achieving an OP/LB was implemented using the generalized estimating equations [15] that was performed to account for possible correlation between contributions of more than 1 cycle from a single patient, with average and peak serum $E_{2}$ levels as primary exposure variables in the model. Pearson's correlation was used to assess the association between peak serum $E_{2}$ levels categorized in percentiles and OP/LB rates. Additionally, a one-sided Fisher's exact test was used to compare the OP/LB rates between the lowest and highest 10th percentiles peak serum E2 levels. In order to determine the predictive ability of average and peak serum $E_{2}$ levels on achieving a clinical pregnancy, a receiver operating characteristic (ROC) curve was created to calculate the cutoff, sensitivity, specificity, and area under the curve (AUC). All statistical analyses were performed using STATA/IC 12.0 software. $P<0.05$ was considered statistically significant.

\section{Results}

A total of 110 artificial FET cycles from 95 patients were analyzed from $1 / 2011$ to $12 / 2014$. Overall pregnancy rate was $56.3 \%$, clinical pregnancy rate was $43.6 \%$, and OP/LB rate was $32.7 \%$. Average number of $E_{2}$ measurements in all cycles following exogenous $\mathrm{E}_{2}$ administration and prior to progesterone supplementation was $4.4 \pm 0.1$ Demographic and cycle characteristics of women are shown in Table 1. 
Table 1 Baseline characteristics in cycles resulting with and without ongoing pregnancy/live birth following an artificial FET cycle

\begin{tabular}{|c|c|c|c|}
\hline Characteristics & Positive OP/LB $(n=36)$ & No OP/LB $(n=74)$ & $P$ value \\
\hline Age at oocyte cryopreservation (years) & $32.1 \pm 0.6$ & $34.3 \pm 0.5$ & 0.01 \\
\hline Max follicle stimulating hormone (IU/L) & $6.4 \pm(5.4-7.8)$ & $5.8(4.9-8.7)$ & 0.79 \\
\hline Gravidity & $1(1-2)$ & $1 \pm(0-2)$ & 0.66 \\
\hline Parity & $1(0-1)$ & $0(0-1)$ & 0.11 \\
\hline Body mass index $\left(\mathrm{kg} / \mathrm{m}^{2}\right)$ & $26.2 \pm 0.9$ & $26.6 \pm 0.7$ & 0.72 \\
\hline Race $(\%)$ & & & 0.64 \\
\hline Caucasian & 38.9 & 28.4 & \\
\hline African American & 22.2 & 20.3 & \\
\hline Hispanic & 11.2 & 12.2 & \\
\hline Other & 27.7 & 39.1 & \\
\hline FET cycle length (days) ${ }^{\mathrm{a}}$ & $23.9 \pm 0.7$ & $23.2 \pm 0.4$ & 0.29 \\
\hline Max endometrial stripe during FET cycle (mm) & $9.6 \pm 0.3$ & $9.5 \pm 0.3$ & 0.85 \\
\hline Route of progesterone administration $(\%)$ & & & 0.49 \\
\hline Intramuscular & 69.4 & 75.7 & \\
\hline Vaginal & 30.6 & 24.3 & \\
\hline Route of E2 administration (\%) & & & 0.33 \\
\hline Transdermal & 44.4 & 41.9 & \\
\hline Intramuscular & 52.8 & 56.8 & \\
\hline Other $^{\mathrm{b}}$ & 2.8 & 1.4 & \\
\hline Vaginal $\mathrm{E}_{2}$ supplementation (\%) & & & 0.54 \\
\hline Yes & 8.3 & 13.5 & \\
\hline No & 91.7 & 86.5 & \\
\hline FET cycle GnRH agonist use (\%) & & & 0.90 \\
\hline GnRH agonist use & 58.3 & 59.5 & \\
\hline No GnRH agonist use & 41.7 & 40.5 & \\
\hline Embryos transferred (number) & $1.8 \pm 0.1$ & $1.8 \pm 0.09$ & 0.98 \\
\hline Day of embryo transfer (\%) & & & 0.008 \\
\hline 5 & 94.4 & 72.9 & \\
\hline 3 & 5.6 & 27.1 & \\
\hline Baseline $E_{2}$ at start of FET cycle (pg/ml) & $35.8 \pm 2.6$ & $32.8 \pm 2.0$ & 0.39 \\
\hline
\end{tabular}

Values are presented as mean \pm standard error of the mean if normally distributed and median with interquartile range if non-normally distributed

FET frozen embryo transfer, $E_{2}$ estradiol, GnRH gonadotropin-releasing hormone agonist

${ }^{\text {a }}$ Denotes days from start of estradiol supplementation to embryo transfer

${ }^{\mathrm{b}} 1$ cycle in cohort without live birth/ongoing pregnancy received estrace vaginally only. One patient in the live birth/ongoing pregnancy cohort received estrace PO only
Age at embryo cryopreservation was significantly lower in cycles resulting in $\mathrm{OP} / \mathrm{LB}$ compared to cycles not resulting in $\mathrm{OP} / \mathrm{LB}(32.1 \pm 0.6$ vs. $34.3 \pm 0.5$ years, respectively, $p=0.01$ ). Additionally, patients who had an OP/LB had more often a day 5 embryo transfer (compared to day 3 embryo transfer) then patients without an OP/LB (94.4\% vs. $73.0 \%$, respectively, $p=<0.01$ ).

Average serum $\mathrm{E}_{2}$ levels were lower in cycles with positive OP/LB outcome compared to cycles with no OP/LB $(234.1 \pm 16.6 \mathrm{pg} / \mathrm{ml}$ vs. $315 \pm 24.8 \mathrm{pg} / \mathrm{ml}$, respectively, $p=0.04)$. As stated above, average age at cryopreservation was lower and percentage of day 5 embryo transfer were higher in cycles that ended up with $\mathrm{OP} / \mathrm{LB}$; however, these variables were not associated with either average or peak $E_{2}$ levels. Hence, these variables were not considered as potential confounders, and were excluded from the regression model when average $E_{2}$ and peak $E_{2}$ levels were analyzed to predict live birth rates. Logistic regression implemented with generalized estimating equations showed that average $\mathrm{E}_{2}$ levels predicted lack of OP/LB rate ( $p=0.04,95 \%$ CI: 0.0001-0.007). Since higher serum average $\mathrm{E}_{2}$ levels were associated with lower likelihood of achieving a clinical pregnancy, we wanted to determine an optimal average serum $\mathrm{E}_{2}$ cutoff level to predict $\mathrm{OP} / \mathrm{LB}$ with good accuracy. For this purpose, an ROC curve was created (Fig. 1) and demonstrated that average serum E2 level of $\geq 330 \mathrm{pg} / \mathrm{mL}$ was associated with no OP/LB 


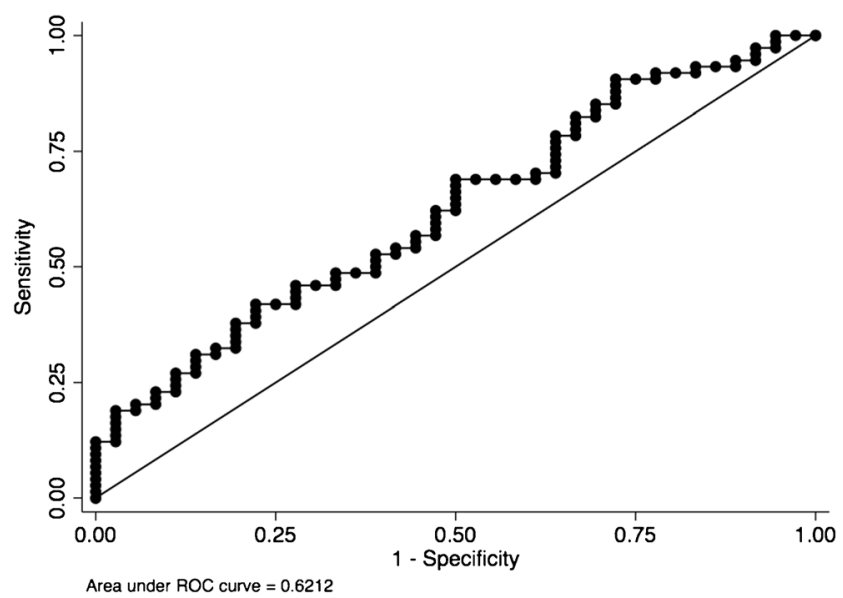

Fig. 1 Receiver operating characteristics curve. Average serum E2 level of $\geq 330 \mathrm{pg} / \mathrm{mL}$ is associated with no ongoing pregnancy/live birth (OP/ LB) with a low sensitivity of $37.8 \%$ but a specificity of $80.6 \%$ and an AUC of 0.62

with a low sensitivity of $37.8 \%$, but a specificity of $80.6 \%$, and an AUC of 0.62 .

There was not a significant difference between peak $\mathrm{E}_{2}$ levels in OP/LB group compared to those without OP/LB $(366.9 \pm 27.7 \mathrm{pg} / \mathrm{ml}$ vs. $459.1 \pm 32.3 \mathrm{pg} / \mathrm{ml}$, respectively, $p=0.19)$. The patients were categorized into three groups according to their peak serum E2 levels: lowest 10th percentile, 11th to 90th percentile, and the highest 10th percentile. Correlation analysis revealed a statistically significant $(p=0.02)$ downward trend in OP/LB rates with increasing peak $\mathrm{E}_{2}$ levels (Fig. 2). Cycles with the highest 10th percentile peak $E_{2}$ levels $(692-1713 \mathrm{pg} / \mathrm{ml})$ had a OP/LB rate of $9.1 \%$ compared to $32.9 \%$ in the 11 th to 90 th percentile (215$689 \mathrm{pg} / \mathrm{ml}$ ) and $54.6 \%$ in the lowest 10th percentile peak $\mathrm{E}_{2}$ levels (135-214 pg/ml). Having a serum estradiol level in the lower 10th percentile was more likely to achieve an OP/LB when compared to having a serum estradiol level in the upper 10th percentile (54.6 vs. $9.1 \%$, respectively, OR: $12, p=0.03$ ).

\section{Discussion}

In this study, we have shown that increasing average serum $E_{2}$ levels during autologous FET cycles are associated with decreasing $\mathrm{OP} / \mathrm{LB}$ rates. We have also shown that $\mathrm{OP} / \mathrm{LB}$ rates decrease from $54 \%$ for peak serum $\mathrm{E}_{2}$ levels below $234 \mathrm{pg} / \mathrm{ml}$ to $9 \%$ for peak serum $\mathrm{E}_{2}$ levels above $692 \mathrm{pg} / \mathrm{ml}$.

Placental implantation is a complex process requiring communication between maternal and fetal tissues [16]. For implantation, placentation, and a healthy subsequent pregnancy outcome, trophoblast cells derived from the placenta must invade through the endometrium, inner third of the myometrium, and eventually remodel the spiral arteries [6]. In the follicular phase, $E_{2}$ is necessary for proper endometrial maturation through induction of endometrial progesterone receptors

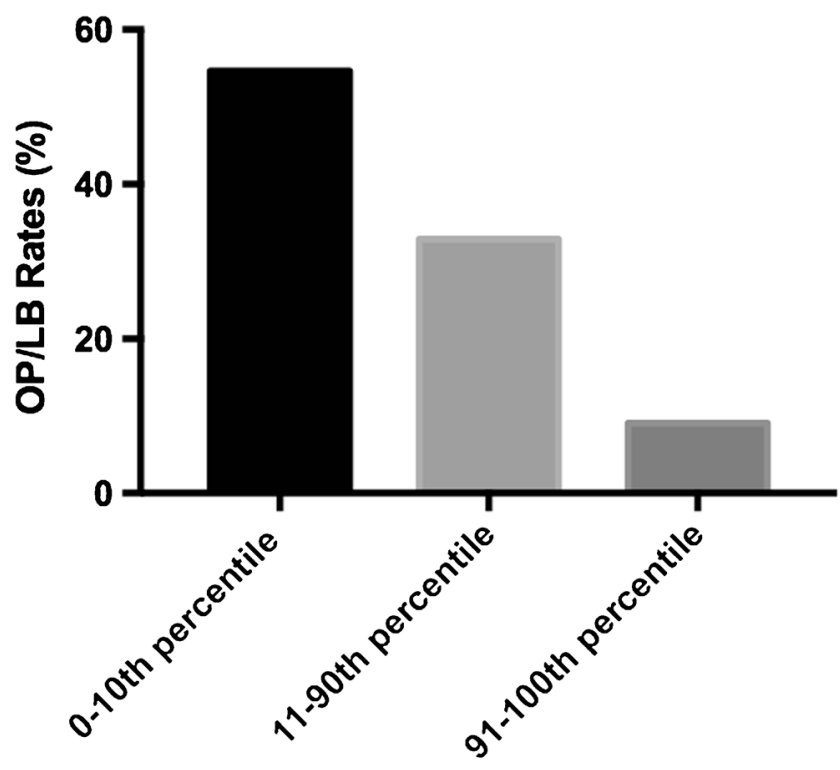

Serum peak E2 levels (pg/ml)

Fig. 2 Correlation between ongoing pregnancy/live birth (OP/LB) rates and peak serum estradiol (E2) levels. Ongoing pregnancy/live birth rates are negatively correlated with increasing peak serum estradiol levels $(p=0.02)$

necessary for implantation [17]. Despite the necessity of $E_{2}$ in the follicular phase for implantation, excess $E_{2}$ during the follicular phase may have a detrimental role. Estradiol has been shown in vitro to cause cell death and inhibit trophoblast invasion in both first-trimester human cytotrophoblast cell line and first-trimester placental explants [18]. In a mouse model, $\mathrm{E}_{2}$ must be held in relatively narrow range for the endometrium to be in a receptive state, and elevated $\mathrm{E}_{2}$ in preparation for a receptive state is associated with altered endometrial expression of genes necessary for implantation [19]. In a baboon model, elevated $\mathrm{E}_{2}$ levels in the first 60 days of pregnancy adversely affected extravillous trophoblast invasion and uterine artery function [20]. Clinically, elevated $\mathrm{E}_{2}$ levels following controlled ovarian hyperstimulation during IVF cycles have been associated with poorer pregnancy rates $[11,12]$ and adverse placenta-related pregnancy outcomes $[13,14]$.

Exogenous $\mathrm{E}_{2}$ administered in artificial FET cycles prior to progesterone supplementation is analogous to the endogenous $\mathrm{E}_{2}$ during the follicular phase in natural cycles. During natural cycles, $\mathrm{E}_{2}$ progressively rises, triggering an LH surge at levels greater than $200 \mathrm{pg} / \mathrm{ml}$ for at least $50 \mathrm{~h}$ [21], and peaks at approximately $300-400 \mathrm{pg} / \mathrm{ml}$ around the time of ovulation followed by an abrupt and steep decline [22]. In our study, higher average $\mathrm{E}_{2}$ levels were found in cycles not resulting in a OP/LB compared to those resulting in an OP/LB despite $\mathrm{E}_{2}$ levels being in the range found during the follicular phase of natural cycles. This may be explained by the timing of $E_{2}$ measurements. $E_{2}$ levels were measured on the day the patient was scheduled to administer exogenous $\mathrm{E}_{2}$, and therefore represented a trough level. Given measurement of trough levels, 
it is likely that the $E_{2}$ levels the endometrium was exposed to during the artificial FET cycles were higher than those measured. In addition, in natural cycles there is an immediate drop in $\mathrm{E}_{2}$ following the LH surge, which does not occur during artificial FET cycles. Although peak levels did not significantly differ between cycles with an OP/LB compared to those without, there was a significant difference when extreme levels were analyzed. In the cycles with the highest 10th percentile peak $E_{2}$ levels, there was a sixfold decrease in OP/LB compared to cycles with the lowest 10th percentile peak $\mathrm{E}_{2}$ levels. The levels obtained in the top 10thpercentile of peak $E_{2}$ levels ranged between 692 and $1713 \mathrm{pg} / \mathrm{ml}$, representing supraphysiologic levels that are not normally seen prior to the rise of progesterone in natural cycles. This suggests that elevated $E_{2}$ levels prior to progesterone stabilization of the endometrium may have detrimental effects on endometrial/ blastocyst synchrony. Interestingly, the lowest 10th percentile of $\mathrm{E}_{2}$ levels ranged between 135 and $214 \mathrm{pg} / \mathrm{ml}$ and resulted in the highest $\mathrm{OP} / \mathrm{LB}$ rates despite being lower than physiologic levels seen during a natural follicular phase. As described above, these levels likely do not represent the true peak $E_{2}$ levels as trough levels were obtained. Additionally, low levels of $E_{2}(<100 \mathrm{pg} / \mathrm{ml})$ in artificial FET donor oocyte cycles have not been associated with adverse pregnancy rates [23].

Our findings are in contrast to a retrospective study by Niu et al. evaluating the predictive value of $E_{2}$ in artificial FET cycles, revealing that elevated $\mathrm{E}_{2}$ was not predictive of pregnancy rates [24]. Differences in our findings may be related to the timing of $E_{2}$ measurements. In the study by Niu et al. [24], prior to progesterone administration, $\mathrm{E}_{2}$ levels were measured only on the day ultrasound was performed and day of progesterone initiation, whereas in our study $\mathrm{E}_{2}$ was measured on average 4.4 times per cycle following the onset of $E_{2}$ administration and prior to the onset of progesterone supplementation. In the above study, $\mathrm{E}_{2}$ levels were separated into three percentiles $(0-25$ th $\%, 25$ th -75 th $\%$, and 75 th-100th $\%)$. The highest $\mathrm{E}_{2}$ levels on the day of progesterone administration in the 75th to 100 th\% range were lower on average $(299 \mathrm{pg} / \mathrm{ml})$ than levels we obtained in our cycles. Another major and important difference was the route of $E_{2}$ administration. Cycles in the present study were administered predominantly with transdermal and intramuscular $\mathrm{E}_{2}$ whereas, in the study by Niu et al. the route of $\mathrm{E}_{2}$ administration was oral. Oral $\mathrm{E}_{2}$ is extensively metabolized by the intestine and liver to estrone, an estrogen with weaker estrogenic activity and weaker binding affinity for estrogen receptors compared to $E_{2}$ [17]. In contrast, transdermal and intramuscular administration of $\mathrm{E}_{2}$ bypass first-pass metabolism and result in less conversion to estrone [17]. It is possible that the endometrium is more sensitive to $\mathrm{E}_{2}$ administered via the intramuscular or transdermal routes compared to $\mathrm{E}_{2}$ administered orally due to less conversion to estrone.

Our findings are also in contrast to findings of Remohi et al. that found no difference in implantation rates in artificial oocyte donation FET cycles with $\mathrm{E}_{2}$ levels measured on the day of progesterone administration. They grouped cycles into $\mathrm{E}_{2}$ levels $<100 \mathrm{pg} / \mathrm{ml}, 100-199 \mathrm{pg} / \mathrm{ml}, 200-299 \mathrm{pg} / \mathrm{ml}, 300$ $399 \mathrm{pg} / \mathrm{ml}$, and $\geq 400 \mathrm{pg} / \mathrm{ml}$ [23]. In this study, patients were desensitized with leuprolide acetate followed by oral estradiol initiated at $2 \mathrm{mg} /$ day. This study also used oral $\mathrm{E}_{2}$, which may account for the discrepant findings. Additionally, the patient population in this study were donor oocyte recipients, whereas, patients in our study were autologous FET recipients.

Despite physiological differences in artificial and natural FET cycles, meta-analyses reveal no significant differences in pregnancy rates [2, 25]. Advantages of artificial FET cycles benefit anovulatory patients and include ability to control the timing of FET cycle start and embryo transfer. Our study suggests that when performing an artificial FET cycle, $\mathrm{E}_{2}$ levels should be monitored and $\mathrm{E}_{2}$ dosage should be adjusted accordingly. Results of this study demonstrate that, ideally, trough peak $\mathrm{E}_{2}$ levels should not reach above approximately $234 \mathrm{pg} / \mathrm{ml}$. In cycles in which peak $\mathrm{E}_{2}$ levels are approaching this level, dosage may be lowered, or alternatively, if levels exceed this threshold, cycle can be canceled and restarted.

In conclusion, elevated $\mathrm{E}_{2}$ levels in artificial autologous FET cycles utilizing intramuscular or transdermal $\mathrm{E}_{2}$ could negatively impact OP/LB rates, possibly due to adverse effect on the endometrium from excess unopposed $E_{2}$ exposure. This is a retrospective study in a single facility, and therefore, larger prospective studies are needed to confirm our results and further elucidate optimal monitoring regimens and $\mathrm{E}_{2}$ levels in artificial autologous FET cycles.

Acknowledgements The authors would like to thank Xiaonan (Nan) Xue, Director, Biostatistics Shared Resource, at Albert Einstein Cancer Center, for her statistical guidance.

\section{References}

1. Sunderam S, Kissin DM, Crawford SB, Folger SG, Jamieson DJ, Warner L, et al. Assisted reproductive technology surveillanceUnited States, 2013. MMWR Surveill Summ. 2015;64(11):1-25. doi:10.15585/mmwr.ss6411a1.

2. Groenewoud ER, Cantineau AE, Kollen BJ, Macklon NS, Cohlen BJ. What is the optimal means of preparing the endometrium in frozen-thawed embryo transfer cycles? A systematic review and meta-analysis. Hum Reprod Update. 2013;19(5):458-70. doi:10. 1093/humupd/dmt030.

3. Shapiro BS, Daneshmand ST, Garner FC, Aguirre M, Hudson C, Thomas S. Evidence of impaired endometrial receptivity after ovarian stimulation for in vitro fertilization: a prospective randomized trial comparing fresh and frozen-thawed embryo transfer in normal responders. Fertil Steril. 2011;96(2):344-8. doi:10.1016/j.fertnstert. 2011.05.050.

4. Check JH, Choe JK, Katsoff D, Summers-Chase D, Wilson C. Controlled ovarian hyperstimulation adversely affects implantation following in vitro fertilization-embryo transfer. J Assist Reprod Genet. 1999;16(8):416-20. 
5. Zhu D, Zhang J, Cao S, Zhang J, Heng BC, Huang M, et al. Vitrified-warmed blastocyst transfer cycles yield higher pregnancy and implantation rates compared with fresh blastocyst transfer cycles - time for a new embryo transfer strategy? Fertil Steril. 2011;95(5):1691-5. doi:10.1016/j.fertnstert.2011.01.022.

6. Pijnenborg R, Vercruysse L, Hanssens M. The uterine spiral arteries in human pregnancy: facts and controversies. Placenta. 2006;27(910):939-58. doi:10.1016/j.placenta.2005.12.006.

7. Fritz R, Jain C, Armant DR. Cell signaling in trophoblast-uterine communication. The International journal of developmental biology. 2014;58(2-4):261-71. doi:10.1387/ijdb.140011da.

8. Villee CA, Hagerman DD. Effects of estradiol on the metabolism of human placenta in vitro. J Biol Chem. 1953;205(2):873-82.

9. Albrecht ED, Aberdeen GW, Pepe GJ. The role of estrogen in the maintenance of primate pregnancy. Am J Obstet Gynecol. 2000;182(2):432-8.

10. Simon C, Cano F, Valbuena D, Remohi J, Pellicer A. Clinical evidence for a detrimental effect on uterine receptivity of high serum oestradiol concentrations in high and normal responder patients. Hum Reprod. 1995;10(9):2432-7.

11. Forman R, Fries N, Testart J, Belaisch-Allart J, Hazout A, Frydman R. Evidence for an adverse effect of elevated serum estradiol concentrations on embryo implantation. Fertil Steril. 1988;49(1):118-22.

12. Arslan M, Bocca S, Arslan EO, Duran HE, Stadtmauer L, Oehninger S. Cumulative exposure to high estradiol levels during the follicular phase of IVF cycles negatively affects implantation. J Assist Reprod Genet. 2007;24(4):111-7. doi: 10.1007/s10815-006-9101-x.

13. Farhi J, Ben-Haroush A, Andrawus N, Pinkas H, Sapir O, Fisch B, et al. High serum oestradiol concentrations in IVF cycles increase the risk of pregnancy complications related to abnormal placentation. Reprod BioMed Online. 2010;21(3):331-7. doi:10.1016/j. rbmo.2010.04.022.

14. Imudia AN, Awonuga AO, Doyle JO, Kaimal AJ, Wright DL, Toth TL, et al. Peak serum estradiol level during controlled ovarian hyperstimulation is associated with increased risk of small for gestational age and preeclampsia in singleton pregnancies after in vitro fertilization. Fertil Steril. 2012;97(6):1374-9. doi:10.1016/j. fertnstert.2012.03.028.
15. Zeger SL, Liang KY, Albert PS. Models for longitudinal data: a generalized estimating equation approach. Biometrics. 1988;44(4):1049-60.

16. Zhang $\mathrm{S}$, Lin $\mathrm{H}$, Kong $\mathrm{S}$, Wang $\mathrm{S}$, Wang $\mathrm{H}$, Wang $\mathrm{H}$, et al. Physiological and molecular determinants of embryo implantation. Mol Asp Med. 2013;34(5):939-80. doi:10.1016/j.mam. 2012.12.011.

17. Paulson RJ. Hormonal induction of endometrial receptivity. Fertil Steril. 2011;96(3):530-5. doi:10.1016/j.fertnstert.2011.07.1097.

18. Patel S, Kilburn B, Imudia A, Armant DR, Skafar DF. Estradiol elicits proapoptotic and antiproliferative effects in human trophoblast cells. Biol Reprod. 2015;93(3):74. doi:10.1095/biolreprod. 115.129114

19. Ma WG, Song H, Das SK, Paria BC, Dey SK. Estrogen is a critical determinant that specifies the duration of the window of uterine receptivity for implantation. Proc Natl Acad Sci U S A. 2003;100(5):2963-8. doi:10.1073/pnas.0530162100.

20. Albrecht ED, Bonagura TW, Burleigh DW, Enders AC, Aberdeen GW, Pepe GJ. Suppression of extravillous trophoblast invasion of uterine spiral arteries by estrogen during early baboon pregnancy. Placenta. 2006;27(4-5):483-90. doi: 10.1016/j.placenta.2005.04.005.

21. Reed BG, Carr BR. The normal menstrual cycle and the control of ovulation. In: De Groot LJ, Chrousos G, Dungan K, Feingold KR, Grossman A, Hershman JM et al., editors. Endotext. South Dartmouth: MDText.com, Inc.; 2000.

22. Hoff JD, Quigley ME, Yen SS. Hormonal dynamics at midcycle: a reevaluation. J Clin Endocrinol Metab. 1983;57(4):792-6. doi:10. 1210/jcem-57-4-792.

23. Remohi J, Ardiles G, Garcia-Velasco JA, Gaitan P, Simon C, Pellicer A. Endometrial thickness and serum oestradiol concentrations as predictors of outcome in oocyte donation. Hum Reprod. 1997;12(10):2271-6.

24. Niu Z, Feng Y, Sun Y, Zhang A, Zhang H. Estrogen level monitoring in artificial frozen-thawed embryo transfer cycles using step-up regime without pituitary suppression: is it necessary? J Exp Clin Assist Reprod. 2008;5:4. doi:10.1186/1743-1050-5-4.

25. Ghobara T, Vandekerckhove P. Cycle regimens for frozen-thawed embryo transfer. The Cochrane database of systematic reviews. 2008;1:CD003414. doi:10.1002/14651858.CD003414.pub2. 